\title{
Bayesian Estimation for Nadarajah-Haghighi Distribution Based on Upper Record Values
}

\author{
Ms. Sana \\ Department of Statistics and Operations Research \\ Aligarh Muslim University, India \\ sanarais233@gmail.com \\ M. Faizan \\ Department of Statistics and Operations Research \\ Aligarh Muslim University, India \\ mdfaizan02@rediffmail.com
}

\begin{abstract}
This paper discusses maximum likelihood and Bayes estimation of the two unknown parameters of Nadarajah and Haghighi distribution based on record values. Different Bayes estimates are derived under squared error, balanced squared error and general entropy loss functions by using Jeffreys' prior information and extension of Jeffreys' prior information. It is observed that the associated posterior distribution appears in an intractable form. So, we have used Tierney and Kadane approximation method to compute these estimates. Finally, numerical computations are presented based on generated record values using $\mathrm{R}$ software.
\end{abstract}

Keywords: Bayesian estimation, Maximum likelihood estimation, Jeffreys' prior information, Extension of Jeffreys' prior information, Loss functions, Tierney and Kadane approximation, Record values.

1. Introduction: Suppose that $\left\{X_{i} ; i \geq 1\right\}$ be a sequence of independent and identically distributed (iid) random variables from an absolutely continuous distribution function $(c d f) F(x)$ and probability density function $(p d f) f(x)$. An observation $X_{j}$ will be called an upper record value if its value exceeds that of all previous observations. Thus, $X_{j}$ is an upper record if $X_{j}>X_{i}$ for every $i<j$. If the sequence $\{U(n) ; n \geq 1\}$ is defined by

$$
U(1)=1, U(n)=\min \left\{j: j>U(n-1), X_{j}>X_{U(n-1)}\right\}
$$

for $n \geq 2$, then the sequence $\left\{R_{n}=X_{U(n)} ; n \geq 1\right\}$ is called the upper record values. The sequence $\{U(n) ; n \geq 1\}$ is called the upper record times.

In a sequence of events, the event value that exceeds all previous values is of particular importance in the scientific and applied fields and so their values are recorded. In sporting events, for example, focus attention is usually on recording results that exceed their predecessor, as the hydrologists usually tend to monitor the higher values of the floods. Also, the meteorologists usually concern with upper and lower record temperatures. For more details on the concept of record values and their application, see, Ahsanullah (2004) and Arnold et al. (1998). The statistical treatment of the record values was introduced for the first time by Chandler (1952). Many studies on record values and their associated 
statistical inference have been done for some distributions by several authors such as Selim (2012) who studied Bayesian estimation of Chen distribution based on record values.

Nadarajah and Haghighi (2011) introduced a new extension of the exponential distribution as an alternative to the gamma, Weibull and the exponentiated exponential distributions. The corresponding $c d f$ and $p d f$ are as follows:

$$
F(\alpha, \lambda)=1-\exp \left\{1-(1+\lambda x)^{\alpha}\right\}, \quad x>0, \alpha, \lambda>0
$$

and

$$
f(\alpha, \lambda)=\alpha \lambda(1+\lambda x)^{\alpha-1} \exp \left\{1-(1+\lambda x)^{\alpha}\right\}, \quad x>0, \alpha, \lambda>0 .
$$

Here $\alpha>0$ and $\lambda>0$ are scale and shape parameters, respectively. Henceforth, Nadarajah and Haghighi distribution will be denoted by $(\mathrm{NH})$ distribution. Singh et al. (2015) discussed classical and Bayesian estimations for $N H$ model under progressive typeII censored data. The maximum likelihood estimators ( $M L E$ 's) and Bayes estimators of the unknown parameters of $\mathrm{NH}$ distribution under progressive type-II censored data with binomial removals have also been obtained by Singh et al. (2014).

Jeffreys' prior exhibits many nice features that make it an attractive reference prior. Jeffreys' prior has the property of being approximately non informative in the sense of Box and Tiao (1973), who motivated Jeffreys' prior by introducing the notion of data translated likelihood. Bernardo (1979) showed that, under certain conditions, Jeffreys' prior is an optimal reference prior in the sense that it maximizes the missing information. Kass (1989, 1990) emphasized that a main feature of Jeffreys' prior is that it is a uniform measure in an information metric, which can be regarded as the natural metric for statistical inference. In addition, for problems involving scale and location parameters, Jeffreys' (1946, 1961), Box and Tiao (1973), Bernardo (1979) and Kass (1990) pointed out that Jeffreys' multivariate prior should be modified as suggested by Jeffreys' (1946, 1961).

Kumar et al. (2017) discussed the Bayes estimators under symmetric and asymmetric loss functions using Markov Chain Monte Carlo (MCMC) technique to compare the performance of the proposed methods based on record values. Gencer and Saraçoğlu (2016) compares the approximate Bayes estimators under different loss functions for parameters of odd Weibull distribution and the Bayes estimators of the parameters of Erlang distribution under different prior distributions have been obtained by Haq and Dey (2011). For detail survey one may refer to Jung and Chung (2018), Pandey and Kumari (2018a, b), Kayal et al. (2016) and Faizan and Sana (2018) amongst others.

The approximate Bayes estimators of the unknown parameters of $\mathrm{NH}$ distribution using Lindley's approximation method based on record values have been obtained by Selim (2018). In this paper, $M L E$ 's and approximate Bayes estimators of the parameters of the $N H$ distribution based on upper record values are obtained under squared error loss (SEL), balance squared error loss $(B S E L)$ and general entropy loss $(G E L)$ functions using Tierney and Kadane (TK) approximation technique with Jeffreys' prior information and extension of Jeffreys' prior information. Moreover, numerical computations using $R$ software are given to illustrate the results.

\section{Estimation}

In this section, we derive the $M L E$ 's of the parameters from record data. We will also study the Bayesian estimation of the two unknown parameters of $\mathrm{NH}$ distribution based on a sample of record values. 


\subsection{Maximum likelihood estimator}

Suppose we observe $m$ upper record values $X_{U(1)}=x_{1}, X_{U(2)}=x_{2}, \ldots, X_{U(m)}=x_{m}$, from $\mathrm{NH}$ distribution with $c d f(1.1)$ and $p d f(1.2)$. The likelihood associated with record data is given by (Ahsanullah, 2004)

$$
L(\alpha, \lambda \mid \underline{x})=f\left(x_{m} ; \alpha, \lambda\right) \prod_{i=1}^{m-1} h\left(x_{i} ; \alpha, \lambda\right)
$$

where

$$
\underline{x}=\left(x_{1}, x_{2}, \ldots, x_{m}\right) \quad \text { and } \quad h\left(x_{i} ; \alpha, \lambda\right)=\frac{f\left(x_{i} ; \alpha, \lambda\right)}{1-F\left(x_{i} ; \alpha, \lambda\right)} .
$$

Substituting (1.1) and (1.2) in (2.1), we get

$$
L(\alpha, \lambda \mid \underline{x})=\alpha^{m} \lambda^{m} \exp \left\{1-\left(1+\lambda x_{m}\right)^{\alpha}\right\} \prod_{i=1}^{m}\left(1+\lambda x_{i}\right)^{\alpha-1}
$$

The log-likelihood function may then be written as

$$
\ln L(\alpha, \lambda \mid \underline{x})=m(\ln \alpha+\ln \lambda)+1-\left(1+\lambda x_{m}\right)^{\alpha}+(\alpha-1) \sum_{i=1}^{m} \ln \left(1+\lambda x_{i}\right) .
$$

Taking derivatives with respect to $\alpha$ and $\lambda$ of (2.3) and equating them to zero, we obtain the likelihood equations for $\alpha$ and $\lambda$ to be

$$
\frac{\partial \ln L(\alpha, \lambda \mid \underline{x})}{\partial \alpha}=\frac{m}{\alpha}-\left(1+\lambda x_{m}\right)^{\alpha} \ln \left(1+\lambda x_{m}\right)+\sum_{i=1}^{m} \ln \left(1+\lambda x_{i}\right)=0
$$

and

$$
\frac{\partial \ln L(\alpha, \lambda \mid \underline{x})}{\partial \lambda}=\frac{m}{\lambda}-\alpha x_{m}\left(1+\lambda x_{m}\right)^{\alpha-1}+(\alpha-1) \sum_{i=1}^{m} \frac{x_{i}}{\left(1+\lambda x_{i}\right)}=0 .
$$

The Equations (2.4) and (2.5) cannot be solved analytically for $\alpha$ and $\lambda$. Therefore, we may use $R$ software (using optim function, see, R Core Team (2018)) to solve these equations and find the MLE's of the unknown parameters $\alpha$ and $\lambda$.

\subsection{Bayesian estimation}

For Bayesian estimation of the parameters $\alpha$ and $\lambda$, prior distributions are needed. If once prior knowledge about the parameter is available, it is suitable to make use of an informative prior but in a situation where one does not have any prior knowledge about the parameter and cannot obtain vital information from experts in this regard, then a noninformative prior will be a suitable alternative to use, (Guure et al., 2013). A commonly used reference prior in Bayesian analysis is Jeffreys' prior. It is obtained by applying Jeffreys' rule, which is to take the prior density to be proportional to the square root of the determinant of the Fisher information matrix (Lavanya and Alexander, 2016). In this study, Jeffreys' priors are use and these are as follows

$$
\begin{aligned}
& \Pi(\alpha) \propto\left[\frac{1}{\alpha}\right] \\
& \Pi(\lambda) \propto\left[\frac{1}{\lambda}\right]
\end{aligned}
$$


The joint prior of parameters are

$$
\Pi(\alpha, \lambda) \propto\left[\frac{1}{\alpha \lambda}\right] .
$$

We propose an extension of Jeffreys' prior information such that,

$$
\Pi(\alpha, \lambda) \propto\left[\frac{1}{\alpha \lambda}\right]^{2 c},
$$

where $c$ is the hyper parameter that is assumed to be non-negative and known. When $c=1$ , we have the standard Jeffreys' prior information and undefined when $c=0$. Since our knowledge on the parameters is limited as a result of which a Jeffreys' prior information approach is employed on both parameters. It is important that one ensures the prior does not significantly influence the final result. If our limited or lack of knowledge influences the results, one may end-up giving wrong interpretation which could affect whatever it is we seek to address.

\subsubsection{Bayes estimation under squared error loss function}

In this section, we obtain Bayes estimates of $\alpha$ and $\lambda$ using SEL and BSEL functions. Let any function of $\alpha$ and $\lambda$ be $u(\alpha, \lambda)=\theta$. We introduce the BSEL function as (Ahmed, 2014)

$$
L(\hat{\theta}-\theta)=\omega\left(\hat{\theta}-\theta_{0}\right)^{2}+(1-\omega)(\hat{\theta}-\theta)^{2},
$$

where $\theta_{0}$ is a known estimator of $\theta$ and $0 \leq \omega \leq 1$. This loss function reduces to the squared error, when $\omega=0$. The Bayes estimate of $\theta$ under the loss $L($.) is obtained to be

$$
\hat{u}_{B S E L}(\alpha, \lambda)=\omega \theta_{0}+(1-\omega) E(\theta \mid \underline{x}) \text {. }
$$

$S E L$ function is a symmetric function and was introduced by Legendre (1805). Let any function of $\alpha$ and $\lambda$ be $u(\alpha, \lambda)=\theta$. The SEL function is as follows:

$$
L(\hat{\theta}-\theta) \propto(\hat{\theta}-\theta)^{2},
$$

where $\hat{\theta}$ is the estimate of the parameter $\theta$. Under the above loss function, the Bayes estimator $\hat{\theta}_{S E L}$ of $\theta$ is given by

$$
\hat{\theta}_{S E L}=\left[E_{\theta}(\theta \mid \underline{x})\right]
$$

where $E_{\theta}$ stands for posterior expectation. In this case, Bayes estimator of $u(\alpha, \lambda)$ under $S E L$ function which is a symmetric loss function is obtained as follows:

$$
\begin{aligned}
\hat{u}_{S E L}(\alpha, \lambda) & =E[u(\alpha, \lambda) \mid \underline{x}] \\
& =\int_{0}^{\infty} \int_{0}^{\infty} u(\alpha, \lambda) \Pi(\alpha, \lambda \mid \underline{x}) d \alpha d \lambda \\
= & \frac{\int_{0}^{\infty} \int_{0}^{\infty} u(\alpha, \lambda) \mathrm{e}^{[l(\alpha, \lambda \mid \underline{x})+\rho(\alpha, \lambda \mid \underline{x})]} d \alpha d \lambda}{\int_{0}^{\infty} \int_{0}^{\infty} \mathrm{e}^{[l(\alpha, \lambda \mid \underline{x})+\rho(\alpha, \lambda \mid \underline{x})]} d \alpha d \lambda},
\end{aligned}
$$


where $l(\alpha, \lambda \mid \underline{x})$ is log-likelihood function and $\rho(\alpha, \lambda \mid \underline{x})$ is logarithm of joint prior distribution.

\subsubsection{Bayes estimation under general entropy loss function}

GEL function is an asymmetric function and was suggested by Calabria and Pulcini (1996). Dey and Liao (1992) have studied about Bayes estimation under GEL function. Let any function of $\alpha$ and $\lambda$ be $u(\alpha, \lambda)=\theta$. The GEL function with parameter $k$ is given by

$$
L(\hat{\theta}-\theta) \propto\left(\frac{\hat{\theta}}{\theta}\right)-k \ln \left(\frac{\hat{\theta}}{\theta}\right)-1,
$$

where $\hat{\theta}$ is the estimate of the parameter $\theta$.

Under the above loss function, the Bayes estimator $\hat{\theta}_{G E}$ of $\theta$ is given by

$$
\hat{\theta}_{G E L}=\left[E_{\theta}\left(\theta^{-k} \mid \underline{x}\right)\right]^{-\frac{1}{k}}
$$

where $E_{\theta}$ stands for posterior expectation. The proper choice for $k$ is a challenging task for an analyst because it reflects the asymmetry of the loss function. In this case, Bayes estimator of $u(\alpha, \lambda)$, under $G E L$ function which is an asymmetric loss function is obtained as follows:

$$
\begin{aligned}
\hat{u}_{G E L}(\alpha, \lambda)=\left[E\left\{[u(\alpha, \lambda)]^{-k} \mid \underline{x}\right\}\right]^{-\frac{1}{k}} \\
=\left[\frac{\left.\int_{0}^{\infty} \int_{0}^{\infty}[u(\alpha, \lambda)]^{-k} \mathrm{e}^{[l(\alpha, \lambda \mid \underline{x})+\rho(\alpha, \lambda \mid \underline{x})]} d \alpha d \lambda\right]^{-\frac{1}{k}}}{\int_{0}^{\infty} \int_{0}^{\infty} \mathrm{e}^{[l(\alpha, \lambda \mid \underline{x})+\rho(\alpha, \lambda \mid \underline{x})]} d \alpha d \lambda} .\right.
\end{aligned}
$$

It is difficult to solve the equations (2.12) and (2.14) in closed form. Because of this reason, the Bayes estimators of the parameters $\alpha$ and $\lambda$ can be obtained using $T K$ approximation method.

\section{Tierney-Kadane approximation}

Tierney and Kadane (1986) is one of the methods to find the approximate value of the mathematical explanations as the ratio of two integrals given in equations (2.12) and (2.14). We consider posterior expectation of $u(\alpha, \lambda)$ with respect to the distribution $\Pi(\alpha, \lambda \mid \underline{x})$ and then assume that

$$
I(x)=\frac{\int_{0}^{\infty} \int_{0}^{\infty} u(\alpha, \lambda) \mathrm{e}^{[l(\alpha, \lambda \mid \underline{x})+\rho(\alpha, \lambda \mid \underline{x})]} d \alpha d \lambda}{\int_{0}^{\infty} \int_{0}^{\infty} \mathrm{e}^{[l(\alpha, \lambda \mid \underline{x})+\rho(\alpha, \lambda \mid \underline{x})]} d \alpha d \lambda},
$$

where $u(\alpha, \lambda)$ is any function of $\alpha$ and $\lambda, l(\alpha, \lambda \mid \underline{x})$ is defined in equation (2.3). $\rho(\alpha, \lambda \mid \underline{x})$ is logarithm joint prior distribution and is defined as follows:

For Jeffreys' prior information, 


$$
\rho(\alpha, \lambda \mid \underline{x})=\ln (\Pi(\alpha, \lambda))=-2 c \ln (\alpha)-2 c \ln (\lambda)
$$

and for extension of Jeffreys' prior information,

$$
\rho(\alpha, \lambda \mid \underline{x})=\ln (\Pi(\alpha, \lambda))=-\ln (\alpha)-\ln (\lambda) .
$$

We can approximate the function $I(x)$ into an explicit expression by applying the $T K$ approximation method. In sequel, we consider the functions defined by

$$
\delta(\alpha, \lambda)=\frac{l(\alpha, \lambda \mid \underline{x})+\rho(\alpha, \lambda \mid \underline{x})}{n}
$$

and

$$
\delta_{\theta}^{*}(\alpha, \lambda)=\delta(\alpha, \lambda)+\frac{\ln u(\alpha, \lambda)}{n} .
$$

Now, we assume that $\delta\left(\hat{\alpha}_{\delta}, \hat{\lambda}_{\delta}\right)$ and $\left(\hat{\alpha}_{\delta^{*}}, \hat{\lambda}_{\delta^{*}}\right)$ maximize the functions $\delta(\alpha, \lambda)$ and $\delta_{\theta}^{*}(\alpha, \lambda)$, respectively.

We then approximate $I(x)$ as

$$
I(x)=\sqrt{\frac{\left|\Sigma_{\theta}^{*}\right|}{|\Sigma|}} \exp \left[n\left\{\delta_{\theta}^{*}\left(\hat{\alpha}_{\delta^{*}}, \hat{\lambda}_{\delta^{*}}\right)-\delta\left(\hat{\alpha}_{\delta}, \hat{\lambda}_{\delta}\right)\right\}\right] .
$$

Here, $|\Sigma|$ and $\left|\Sigma_{\theta}^{*}\right|$ denote the determinants of negative inverse hessian of $\delta(\alpha, \lambda)$ and $\delta_{\theta}^{*}(\alpha, \lambda)$, respectively. $|\Sigma|$ and $\left|\Sigma_{\theta}^{*}\right|$ are defined as follows:

$$
|\Sigma|=\left[\frac{\partial^{2} \delta}{\partial \alpha^{2}} \frac{\partial^{2} \delta}{\partial \lambda^{2}}-\frac{\partial^{2} \delta}{\partial \alpha \partial \lambda} \frac{\partial^{2} \delta}{\partial \lambda \partial \alpha}\right]^{-1}
$$

and

$$
\left|\Sigma_{\theta}^{*}\right|=\left[\frac{\partial^{2} \delta_{\theta}^{*}}{\partial \alpha^{2}} \frac{\partial^{2} \delta_{\theta}^{*}}{\partial \lambda^{2}}-\frac{\partial^{2} \delta_{\theta}^{*}}{\partial \alpha \partial \lambda} \frac{\partial^{2} \delta_{\theta}^{*}}{\partial \lambda \partial \alpha}\right]^{-1}
$$

Next, we observe that

$$
\delta(\alpha, \lambda)=\frac{1}{n}\left[m \ln (\alpha)+m \ln (\lambda)+1-\left(1+\lambda x_{m}\right)^{\alpha}+(\alpha-1) \sum_{i=1}^{m} \ln \left(1+\lambda x_{i}\right)-2 c \ln (\alpha)-2 c \ln (\lambda)\right] .
$$

Now, we note that

$$
\frac{\partial \delta}{\partial \alpha}=\frac{1}{n}\left[\frac{m}{\alpha}-\left(1+\lambda x_{m}\right)^{\alpha} \ln \left(1+\lambda x_{m}\right)+\sum_{i=1}^{m} \ln \left(1+\lambda x_{i}\right)-\frac{2 c}{\alpha}\right]
$$

and

$$
\frac{\partial \delta}{\partial \lambda}=\frac{1}{n}\left[\frac{m}{\lambda}-\alpha x_{m}\left(1+\lambda x_{m}\right)^{\alpha-1}+(\alpha-1) \sum_{i=1}^{m} \frac{x_{i}}{\left(1+\lambda x_{i}\right)}-\frac{2 c}{\lambda}\right]
$$

Likewise the corresponding second-order derivatives are obtained by 


$$
\begin{array}{r}
\frac{\partial^{2} \delta}{\partial \alpha^{2}}=\frac{1}{n}\left[-\frac{m}{\alpha^{2}}-\left(1+\lambda x_{m}\right)^{\alpha}\left(\ln \left(1+\lambda x_{m}\right)\right)^{2}+\frac{2 c}{\alpha^{2}}\right], \\
\frac{\partial^{2} \delta}{\partial \alpha \partial \lambda}=\frac{\partial^{2} \delta}{\partial \lambda \partial \alpha}=\frac{1}{n}\left[\sum_{i=1}^{m} \frac{x_{i}}{\left(1+\lambda x_{i}\right)}-x_{m}\left(1+\lambda x_{m}\right)^{\alpha-1}-\alpha x_{m}\left(1+\lambda x_{m}\right)^{\alpha-1} \ln \left(1+\lambda x_{m}\right)\right]
\end{array}
$$

and

$$
\frac{\partial^{2} \delta}{\partial \lambda^{2}}=\frac{1}{n}\left[-\frac{m}{\lambda^{2}}-\alpha(\alpha-1) x_{m}^{2}\left(1+\lambda x_{m}\right)^{\alpha-2}-(\alpha-1) \sum_{i=1}^{m} \frac{x_{i}^{2}}{\left(1+\lambda x_{i}\right)^{2}}+\frac{2 c}{\lambda^{2}}\right]
$$

\subsection{Tierney-Kadane Bayes estimator of $u(\alpha, \lambda)$ under squared error loss function} Bayes estimator of $u(\alpha, \lambda)$ under SEL function is defined as follows:

$$
\begin{aligned}
& \hat{u}_{\theta_{S E L}}(\alpha, \lambda)=E[u(\alpha, \lambda) \mid \underline{x}] \\
& =\sqrt{\frac{\left|\sum_{\theta_{S E L}}^{*}\right|}{|\Sigma|}} \exp \left[n\left\{\delta_{\theta_{S E L}}^{*}\left(\hat{\alpha}_{\delta^{*}}, \hat{\lambda}_{\delta^{*}}\right)-\delta\left(\hat{\alpha}_{\delta}, \hat{\lambda}_{\delta}\right)\right\}\right]
\end{aligned}
$$

where

$$
\delta_{\theta_{S E L}}^{*}(\alpha, \lambda)=\delta(\alpha, \lambda)+\frac{\ln u(\alpha, \lambda)}{n} .
$$

Bayes estimators for parameters $\alpha$ and $\lambda$, using equation (3.12) under $S E L$ functions are obtained as follows:

i) If $u(\alpha, \lambda)=\alpha$, then

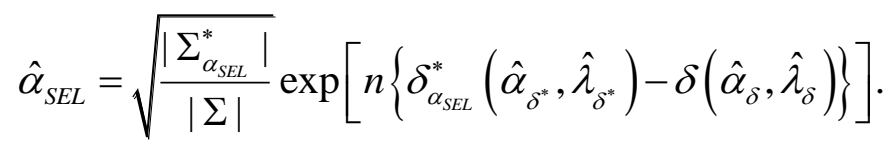

In order to compute $\left|\Sigma_{\alpha_{S E L}}^{*}\right|$, we first obtain the following expressions

$$
\begin{aligned}
& \frac{\partial^{2} \delta^{*}}{\partial \alpha^{2}}=\frac{\partial^{2} \delta}{\partial \alpha^{2}}-\frac{1}{n \alpha^{2}} \\
& \frac{\partial^{2} \delta^{*}}{\partial \lambda^{2}}=\frac{\partial^{2} \delta}{\partial \lambda^{2}} \\
& \frac{\partial^{2} \delta^{*}}{\partial \alpha \partial \lambda}=\frac{\partial^{2} \delta}{\partial \lambda \partial \alpha}
\end{aligned}
$$

ii) If $u(\alpha, \lambda)=\lambda$, then

$$
\hat{\lambda}_{S E L}=\sqrt{\frac{\left|\sum_{\lambda_{S E L}}^{*}\right|}{|\Sigma|}} \exp \left[n\left\{\delta_{\lambda_{S E L}}^{*}\left(\hat{\alpha}_{\delta^{*}}, \hat{\lambda}_{\delta^{*}}\right)-\delta\left(\hat{\alpha}_{\delta}, \hat{\lambda}_{\delta}\right)\right\}\right] .
$$

In order to compute $\left|\Sigma_{\lambda_{\text {SEL }}}^{*}\right|$, we first obtain the following expressions 


$$
\begin{aligned}
\frac{\partial^{2} \delta^{*}}{\partial \alpha^{2}} & =\frac{\partial^{2} \delta}{\partial \alpha^{2}} \\
\frac{\partial^{2} \delta^{*}}{\partial \lambda^{2}} & =\frac{\partial^{2} \delta}{\partial \lambda^{2}}-\frac{1}{n \lambda^{2}} \\
\frac{\partial^{2} \delta^{*}}{\partial \alpha \partial \lambda} & =\frac{\partial^{2} \delta}{\partial \lambda \partial \alpha} .
\end{aligned}
$$

Similarly, we can compute the corresponding Bayes estimates of unknown parameters $\alpha$ and $\lambda$ under the BSEL function.

\subsection{Tierney-Kadane Bayes estimator of $u(\alpha, \lambda)$ under general entropy loss}

\section{function}

Bayes estimator of $u(\alpha, \lambda)$ under $G E L$ function is defined as follows:

$$
\begin{aligned}
\hat{u}_{\theta_{G L L}}(\alpha, \lambda) & =\left[E\left\{[u(\alpha, \lambda)]^{-k} \mid \underline{x}\right\}\right]^{-\frac{1}{k}} \\
& =\left[\sqrt{\frac{\left|\Sigma_{\theta_{G E L}}^{*}\right|}{|\Sigma|}} \exp \left[n\left\{\delta_{\theta_{G E L}}^{*}\left(\hat{\alpha}_{\delta^{*}}, \hat{\lambda}_{\delta^{*}}\right)-\delta\left(\hat{\alpha}_{\delta}, \hat{\lambda}_{\delta}\right)\right\}\right]\right]^{-\frac{1}{k}},
\end{aligned}
$$

where

$$
\delta_{\theta_{G E L}}^{*}(\alpha, \lambda)=\delta(\alpha, \lambda)+\frac{\ln [u(\alpha, \lambda)]^{-k}}{n} .
$$

Bayes estimators for parameters $\alpha$ and $\lambda$ using equation (3.15) under GEL functions are obtained as follows:

i) If $u(\alpha, \lambda)=\alpha^{-k}$, then

$$
\hat{\alpha}_{G E L}=\left[\sqrt{\frac{\left|\Sigma_{\alpha_{G E L}}^{*}\right|}{|\Sigma|}} \exp \left[n\left\{\delta_{\alpha_{G L L}}^{*}\left(\hat{\alpha}_{\delta^{*}}, \hat{\lambda}_{\delta^{*}}\right)-\delta\left(\hat{\alpha}_{\delta}, \hat{\lambda}_{\delta}\right)\right\}\right]\right]^{-\frac{1}{k}} .
$$

In order to compute $\left|\Sigma_{\alpha_{G E L}}^{*}\right|$, we first obtain the following expressions

$$
\begin{aligned}
\frac{\partial^{2} \delta^{*}}{\partial \alpha^{2}} & =\frac{\partial^{2} \delta}{\partial \alpha^{2}}+\frac{k}{n} \alpha^{-2} \\
\frac{\partial^{2} \delta^{*}}{\partial \lambda^{2}} & =\frac{\partial^{2} \delta}{\partial \lambda^{2}} \\
\frac{\partial^{2} \delta^{*}}{\partial \alpha \partial \lambda} & =\frac{\partial^{2} \delta}{\partial \lambda \partial \alpha}
\end{aligned}
$$

ii) If $u(\alpha, \lambda)=\lambda^{-k}$, then

$$
\hat{\lambda}_{G E L}=\left[\sqrt{\frac{\left|\Sigma_{\lambda_{G E L}}^{*}\right|}{|\Sigma|}} \exp \left[n\left\{\delta_{\lambda_{G E L}}^{*}\left(\hat{\alpha}_{\delta^{*}}, \hat{\lambda}_{\delta^{*}}\right)-\delta\left(\hat{\alpha}_{\delta}, \hat{\lambda}_{\delta}\right)\right\}\right]\right]^{-\frac{1}{k}} .
$$

In order to compute $\left|\Sigma_{\lambda_{\text {GEL }}}^{*}\right|$, we first obtain the following expressions 


$$
\begin{aligned}
\frac{\partial^{2} \delta^{*}}{\partial \alpha^{2}} & =\frac{\partial^{2} \delta}{\partial \alpha^{2}} \\
\frac{\partial^{2} \delta^{*}}{\partial \lambda^{2}} & =\frac{\partial^{2} \delta}{\partial \lambda^{2}}+\frac{k}{n} \lambda^{-2} \\
\frac{\partial^{2} \delta^{*}}{\partial \alpha \partial \lambda} & =\frac{\partial^{2} \delta}{\partial \lambda \partial \alpha} .
\end{aligned}
$$

\section{Numerical computations}

In order to illustrate the usefulness of the inference procedures discussed in the previous sections, we generate five sets of record values of sizes 50, 100, 150, 200 and 250 from $N H(3,2)$ distribution as shown in Table 1 . The percentage errors $(P E)$ are computed to assess the performance of the estimators by formula as

$$
P E=\frac{\mid \text { estimate value }- \text { exact value } \mid}{\mid \text { exact value } \mid} \times 100 \% \text {. }
$$

Taking loss parameter $k= \pm 0.6$ and \pm 1.6 , the Bayesian estimates of the parameters are derived with respect to three loss functions, SEL, BSEL and GEL functions. Also, we have considered the values of Jeffreys' extension prior $c=3.5,4.5$ and 5.5. The choice of the extension of Jeffreys' prior information is subjective since it is used to consider the proportion in which one will prefer the prior to influence the posterior density function. The Bayes estimator of $\alpha$ and $\lambda$ under SEL and BSEL function using extension Jeffreys' prior information are shown in the Table 2. The MLE's calculated are also given in the same table. The Bayes estimator of $\alpha$ and $\lambda$ under SEL and BSEL loss function using Jeffreys' prior information are shown in the Table 4. Moreover, The Bayes estimator of $\alpha$ and $\lambda$ under GEL function using extension Jeffreys' prior information and Jeffreys' prior information are shown in the Table 3 and Table 5. Also, the PE is shown along with the estimators.

Table 1. Generated upper record values for different sample sizes.

\begin{tabular}{cccccccccc}
\hline $\mathrm{n}$ & $\mathrm{x}_{1}$ & $\mathrm{x}_{2}$ & $\mathrm{x}_{3}$ & $\mathrm{x}_{4}$ & $\mathrm{x}_{5}$ & $\mathrm{x}_{6}$ & $\mathrm{x}_{7}$ & $\mathrm{x}_{8}$ & $\mathrm{x}_{9}$ \\
\hline 20 & 0.07 & 0.29 & 0.38 & 0.49 & & & & & \\
30 & 0.11 & 0.33 & 0.41 & 0.43 & & & & & \\
10 & 0.12 & 0.17 & 0.19 & 0.2 & 0.24 & 0.3 & 0.42 & & \\
50 & 0.05 & 0.06 & 0.25 & 0.3 & 0.32 & 0.33 & 0.37 & 0.57 & \\
40 & 0.01 & 0.02 & 0.05 & 0.07 & 0.35 & 0.36 & 0.37 & 0.39 & 0.46 \\
\hline
\end{tabular}


Table 2. MLE's and Bayes estimates using extension Jeffreys' prior information for $S E L$ and BSEL function.

\begin{tabular}{|c|c|c|c|c|c|c|c|c|c|c|c|c|c|}
\hline \multirow[t]{4}{*}{$\mathrm{n}$} & \multicolumn{2}{|c|}{ MLE } & \multirow[t]{4}{*}{$c$} & \multicolumn{10}{|c|}{ TK } \\
\hline & \multirow[b]{3}{*}{$\widehat{\alpha}_{M L E}$} & \multirow[b]{3}{*}{$\hat{\lambda}_{M L E}$} & & \multicolumn{2}{|c|}{ SEL } & \multicolumn{8}{|c|}{ BSEL } \\
\hline & & & & \multirow[b]{2}{*}{$\hat{\alpha}_{S E L}$} & \multirow[b]{2}{*}{$\hat{\lambda}_{S E L}$} & \multicolumn{2}{|c|}{$w=0.2$} & \multicolumn{2}{|c|}{$w=0.4$} & \multicolumn{2}{|c|}{$w=0.6$} & \multicolumn{2}{|c|}{$w=0.8$} \\
\hline & & & & & & $\widehat{\alpha}_{B S E L}$ & $\widehat{\lambda}_{B S E L}$ & $\hat{\alpha}_{B S E L}$ & $\hat{\lambda}_{B S E L}$ & $\widehat{\alpha}_{B S E L}$ & $\hat{\lambda}_{B S E L}$ & $\hat{\alpha}_{B S E L}$ & $\widehat{\lambda}_{B S E L}$ \\
\hline \multirow[t]{3}{*}{50} & \multirow[t]{3}{*}{$\begin{array}{l}2.85 \\
(4.9 \%)\end{array}$} & \multirow[t]{3}{*}{$\begin{array}{l}2.18 \\
(8.8 \%)\end{array}$} & 3.5 & $\begin{array}{l}3.13 \\
(4.2 \%)\end{array}$ & $\begin{array}{l}2.21 \\
(10 \%)\end{array}$ & $\begin{array}{l}3.10 \\
(3.4 \%)\end{array}$ & $\begin{array}{l}2.16 \\
(8.2 \%)\end{array}$ & $\begin{array}{l}3.08 \\
(2.5 \%)\end{array}$ & $\begin{array}{l}2.12 \\
(6.2 \%)\end{array}$ & $\begin{array}{l}3.05 \\
(1.7 \%)\end{array}$ & $\begin{array}{l}2.08 \\
(4.1 \%)\end{array}$ & $\begin{array}{l}3.03 \\
(0.8 \%)\end{array}$ & $\begin{array}{l}2.04 \\
(2.1 \%)\end{array}$ \\
\hline & & & 4.5 & $\begin{array}{l}3.06 \\
(2.2 \%)\end{array}$ & $\begin{array}{l}2.19 \\
(5.9 \%)\end{array}$ & $\begin{array}{l}3.05 \\
(1.7 \%)\end{array}$ & $\begin{array}{l}2.09 \\
(4.7 \%)\end{array}$ & $\begin{array}{l}3.03 \\
(1.3 \%)\end{array}$ & $\begin{array}{l}2.07 \\
(3.6 \%)\end{array}$ & $\begin{array}{l}3.03 \\
(0.9 \%)\end{array}$ & $\begin{array}{l}2.05 \\
(2.4 \%)\end{array}$ & $\begin{array}{l}3.01 \\
(0.4 \%)\end{array}$ & $\begin{array}{l}2.02 \\
(1.2 \%)\end{array}$ \\
\hline & & & 5.5 & $\begin{array}{l}3.04 \\
(1.2 \%)\end{array}$ & $\begin{array}{l}2.08 \\
(4.0 \%)\end{array}$ & $\begin{array}{l}3.03 \\
(0.9 \%)\end{array}$ & $\begin{array}{l}2.06 \\
(3.2 \%)\end{array}$ & $\begin{array}{l}3.02 \\
(0.7 \%)\end{array}$ & $\begin{array}{l}2.05 \\
(2.4 \%)\end{array}$ & $\begin{array}{l}3.01 \\
(0.5 \%)\end{array}$ & $\begin{array}{l}2.03 \\
(1.6 \%)\end{array}$ & $\begin{array}{l}3.01 \\
(0.2 \%)\end{array}$ & $\begin{array}{l}2.07 \\
(0.8 \%)\end{array}$ \\
\hline \multirow[t]{3}{*}{100} & \multirow[t]{3}{*}{$\begin{array}{l}2.79 \\
(7.1 \%)\end{array}$} & \multirow[t]{3}{*}{$\begin{array}{l}2.25 \\
(12 \%)\end{array}$} & 3.5 & $\begin{array}{l}3.02 \\
(0.7 \%)\end{array}$ & $\begin{array}{l}2.05 \\
(2.3 \%)\end{array}$ & $\begin{array}{l}3.08 \\
(0.6 \%)\end{array}$ & $\begin{array}{l}2.04 \\
(1.8 \%)\end{array}$ & $\begin{array}{l}3.01 \\
(0.5 \%)\end{array}$ & $\begin{array}{l}2.03 \\
(1.4 \%)\end{array}$ & $\begin{array}{l}3.01 \\
(0.3 \%)\end{array}$ & $\begin{array}{l}2.02 \\
(0.9 \%)\end{array}$ & $\begin{array}{l}3.01 \\
(0.2 \%)\end{array}$ & $\begin{array}{l}2.01 \\
(0.5 \%)\end{array}$ \\
\hline & & & 4.5 & $\begin{array}{l}3.02 \\
(0.5 \%)\end{array}$ & $\begin{array}{l}2.04 \\
(1.9 \%)\end{array}$ & $\begin{array}{l}3.01 \\
(0.4 \%)\end{array}$ & $\begin{array}{l}2.03 \\
(1.5 \%)\end{array}$ & $\begin{array}{l}3.01 \\
(0.3 \%)\end{array}$ & $\begin{array}{l}2.02 \\
(1.2 \%)\end{array}$ & $\begin{array}{l}3.01 \\
(0.2 \%)\end{array}$ & $\begin{array}{l}2.04 \\
(0.8 \%)\end{array}$ & $\begin{array}{l}3.01 \\
(0.1 \%)\end{array}$ & $\begin{array}{l}2.01 \\
(0.4 \%)\end{array}$ \\
\hline & & & 5.5 & $\begin{array}{l}3.01 \\
(0.5 \%)\end{array}$ & $\begin{array}{l}2.03 \\
(1.6 \%)\end{array}$ & $\begin{array}{l}3.01 \\
(0.3 \%)\end{array}$ & $\begin{array}{l}2.02 \\
(1.3 \%)\end{array}$ & $\begin{array}{l}3.01 \\
(0.2 \%)\end{array}$ & $\begin{array}{l}2.02 \\
(0.9 \%)\end{array}$ & $\begin{array}{l}3.01 \\
(0.1 \%)\end{array}$ & $\begin{array}{l}2.01 \\
(0.7 \%)\end{array}$ & $\begin{array}{l}3.00 \\
(0.1 \%)\end{array}$ & $\begin{array}{l}2.01 \\
(0.3 \%)\end{array}$ \\
\hline \multirow[t]{3}{*}{150} & \multirow[t]{3}{*}{$\begin{array}{l}3.09 \\
(3.0 \%)\end{array}$} & \multirow[t]{3}{*}{$\begin{array}{l}1.93 \\
(3.4 \%)\end{array}$} & 3.5 & $\begin{array}{l}3.03 \\
(1.0 \%)\end{array}$ & $\begin{array}{l}2.06 \\
(3.1 \%)\end{array}$ & $\begin{array}{l}3.02 \\
(0.8 \%)\end{array}$ & $\begin{array}{l}2.05 \\
(2.5 \%)\end{array}$ & $\begin{array}{l}3.02 \\
(0.6 \%)\end{array}$ & $\begin{array}{l}2.04 \\
(1.9 \%)\end{array}$ & $\begin{array}{l}3.01 \\
(0.4 \%)\end{array}$ & $\begin{array}{l}2.02 \\
(1.3 \%)\end{array}$ & $\begin{array}{l}3.01 \\
(0.2 \%)\end{array}$ & $\begin{array}{l}2.01 \\
(0.6 \%)\end{array}$ \\
\hline & & & 4.5 & $\begin{array}{l}3.02 \\
(0.6 \%)\end{array}$ & $\begin{array}{l}2.05 \\
(2.4 \%)\end{array}$ & $\begin{array}{l}3.01 \\
(0.5 \%)\end{array}$ & $\begin{array}{l}2.04 \\
(1.9 \%)\end{array}$ & $\begin{array}{l}3.01 \\
(0.4 \%)\end{array}$ & $\begin{array}{l}2.03 \\
(1.5 \%)\end{array}$ & $\begin{array}{l}3.01 \\
(0.3 \%)\end{array}$ & $\begin{array}{l}2.02 \\
(0.9 \%)\end{array}$ & $\begin{array}{l}3.01 \\
(0.1 \%)\end{array}$ & $\begin{array}{l}2.01 \\
(0.5 \%)\end{array}$ \\
\hline & & & 5.5 & $\begin{array}{l}3.01 \\
(0.3 \%)\end{array}$ & $\begin{array}{l}2.04 \\
(1.9 \%)\end{array}$ & $\begin{array}{l}3.01 \\
(0.3 \%)\end{array}$ & $\begin{array}{l}2.03 \\
(1.5 \%)\end{array}$ & $\begin{array}{l}3.01 \\
(0.2 \%)\end{array}$ & $\begin{array}{l}2.02 \\
(1.2 \%)\end{array}$ & $\begin{array}{l}3.01 \\
(0.1 \%)\end{array}$ & $\begin{array}{l}2.02 \\
(0.8 \%)\end{array}$ & $\begin{array}{l}3.00 \\
(0.1 \%)\end{array}$ & $\begin{array}{l}2.01 \\
(0.4 \%)\end{array}$ \\
\hline \multirow[t]{3}{*}{200} & \multirow[t]{3}{*}{$\begin{array}{l}3.01 \\
(0.1 \%)\end{array}$} & \multirow[t]{3}{*}{$\begin{array}{l}1.89 \\
(5.5 \%)\end{array}$} & 3.5 & $\begin{array}{l}3.20 \\
(6.7 \%)\end{array}$ & $\begin{array}{l}2.32 \\
(16 \%)\end{array}$ & $\begin{array}{l}3.16 \\
(5.4 \%)\end{array}$ & $\begin{array}{l}2.26 \\
(12 \%)\end{array}$ & $\begin{array}{l}3.12 \\
(4.0 \%)\end{array}$ & $\begin{array}{l}2.19 \\
(9.6 \%)\end{array}$ & $\begin{array}{l}3.08 \\
(2.7 \%)\end{array}$ & $\begin{array}{l}2.19 \\
(6.4 \%)\end{array}$ & $\begin{array}{l}3.04 \\
(1.3 \%)\end{array}$ & $\begin{array}{l}2.06 \\
(3.2 \%)\end{array}$ \\
\hline & & & 4.5 & $\begin{array}{l}3.09 \\
(3.2 \%)\end{array}$ & $\begin{array}{l}2.16 \\
(7.8 \%)\end{array}$ & $\begin{array}{l}3.08 \\
(2.5 \%)\end{array}$ & $\begin{array}{l}2.12 \\
(6.2 \%)\end{array}$ & $\begin{array}{l}3.06 \\
(1.9 \%)\end{array}$ & $\begin{array}{l}2.09 \\
(4.7 \%)\end{array}$ & $\begin{array}{l}3.04 \\
(1.3 \%)\end{array}$ & $\begin{array}{l}2.06 \\
(3.1 \%)\end{array}$ & $\begin{array}{l}3.02 \\
(0.6 \%)\end{array}$ & $\begin{array}{l}2.03 \\
(1.6 \%)\end{array}$ \\
\hline & & & 5.5 & $\begin{array}{l}3.06 \\
(1.9 \%)\end{array}$ & $\begin{array}{l}2.10 \\
(5.0 \%)\end{array}$ & $\begin{array}{l}3.04 \\
(1.5 \%)\end{array}$ & $\begin{array}{l}2.08 \\
(4.0 \%)\end{array}$ & $\begin{array}{l}3.03 \\
(1.1 \%)\end{array}$ & $\begin{array}{l}2.06 \\
(3.0 \%)\end{array}$ & $\begin{array}{l}3.02 \\
(0.7 \%)\end{array}$ & $\begin{array}{l}2.04 \\
(2.0 \%)\end{array}$ & $\begin{array}{l}3.01 \\
(0.4 \%)\end{array}$ & $\begin{array}{l}2.02 \\
(1.0 \%)\end{array}$ \\
\hline \multirow[t]{3}{*}{250} & \multirow[t]{3}{*}{$\begin{array}{l}2.84 \\
(5.2 \%)\end{array}$} & \multirow[t]{3}{*}{$\begin{array}{l}2.03 \\
(1.5 \%)\end{array}$} & 3.5 & $\begin{array}{l}3.03 \\
(1.1 \%)\end{array}$ & $\begin{array}{l}2.06 \\
(3.1 \%)\end{array}$ & $\begin{array}{l}3.03 \\
(0.9 \%)\end{array}$ & $\begin{array}{l}2.05 \\
(2.5 \%)\end{array}$ & $\begin{array}{l}3.02 \\
(0.9 \%)\end{array}$ & $\begin{array}{l}2.04 \\
(1.9 \%)\end{array}$ & $\begin{array}{l}3.01 \\
(0.5 \%)\end{array}$ & $\begin{array}{l}2.02 \\
(1.2 \%)\end{array}$ & $\begin{array}{l}3.01 \\
(0.2 \%)\end{array}$ & $\begin{array}{l}2.01 \\
(0.6 \%)\end{array}$ \\
\hline & & & 4.5 & $\begin{array}{l}3.03 \\
(0.9 \%)\end{array}$ & $\begin{array}{l}2.05 \\
(2.6 \%)\end{array}$ & $\begin{array}{l}3.02 \\
(0.7 \%)\end{array}$ & $\begin{array}{l}2.04 \\
(2.0 \%)\end{array}$ & $\begin{array}{l}3.02 \\
(0.5 \%)\end{array}$ & $\begin{array}{l}2.03 \\
(1.5 \%)\end{array}$ & $\begin{array}{l}3.01 \\
(0.4 \%)\end{array}$ & $\begin{array}{l}2.02 \\
(1.0 \%)\end{array}$ & $\begin{array}{l}3.01 \\
(0.2 \%)\end{array}$ & $\begin{array}{l}2.01 \\
(0.5 \%)\end{array}$ \\
\hline & & & 5.5 & $\begin{array}{l}3.02 \\
(0.7 \%)\end{array}$ & $\begin{array}{l}2.04 \\
(2.2 \%)\end{array}$ & $\begin{array}{l}3.02 \\
(0.6 \%)\end{array}$ & $\begin{array}{l}2.03 \\
(1.7 \%)\end{array}$ & $\begin{array}{l}3.01 \\
(0.4 \%)\end{array}$ & $\begin{array}{l}2.03 \\
(1.3 \%)\end{array}$ & $\begin{array}{l}3.01 \\
(0.3 \%)\end{array}$ & $\begin{array}{l}2.02 \\
(0.9 \%)\end{array}$ & $\begin{array}{l}3.00 \\
(0.1 \%)\end{array}$ & $\begin{array}{l}2.01 \\
(0.4 \%)\end{array}$ \\
\hline
\end{tabular}


Table 3. Bayes estimates using extension Jeffreys' prior information for GEL function.

\begin{tabular}{|c|c|c|c|c|c|c|c|c|c|c|}
\hline \multirow[t]{4}{*}{$n$} & \multirow[t]{4}{*}{$m$} & \multirow[t]{4}{*}{$c$} & \multicolumn{8}{|c|}{$\mathrm{TK}$} \\
\hline & & & \multicolumn{8}{|c|}{ GSEL } \\
\hline & & & $\hat{\alpha}_{G E L}$ & $\widehat{\lambda}_{G E L}$ & $\hat{\alpha}_{G E L}$ & $\hat{\lambda}_{G E L}$ & $\hat{\alpha}_{G E L}$ & $\widehat{\lambda}_{G E L}$ & $\hat{\alpha}_{G E L}$ & $\widehat{\lambda}_{G E L}$ \\
\hline & & & \multicolumn{2}{|c|}{$k=0.6$} & \multicolumn{2}{|c|}{$k=-0.6$} & \multicolumn{2}{|c|}{$k=1.6$} & \multicolumn{2}{|c|}{$k=-1.6$} \\
\hline \multirow[t]{3}{*}{50} & \multirow[t]{3}{*}{7} & 3.5 & $\begin{array}{c}3.12 \\
(3.93 \%)\end{array}$ & $\begin{array}{c}2.18 \\
(8.82 \%)\end{array}$ & $\begin{array}{c}3.12 \\
(4.12 \%)\end{array}$ & $\begin{array}{c}2.19 \\
(9.87 \%)\end{array}$ & $\begin{array}{c}3.11 \\
(3.78 \%)\end{array}$ & $\begin{array}{c}2.16 \\
(8.14 \%)\end{array}$ & $\begin{array}{c}3.13 \\
(4.30 \%)\end{array}$ & $\begin{array}{c}2.22 \\
(11.04 \%)\end{array}$ \\
\hline & & 4.5 & $\begin{array}{c}3.06 \\
(2.08 \%)\end{array}$ & $\begin{array}{c}2.11 \\
(5.40 \%)\end{array}$ & $\begin{array}{c}3.06 \\
(2.13 \%)\end{array}$ & $\begin{array}{c}2.12 \\
(5.78 \%)\end{array}$ & $\begin{array}{c}3.06 \\
(2.04 \%)\end{array}$ & $\begin{array}{c}2.10 \\
(5.14 \%)\end{array}$ & $\begin{array}{c}3.07 \\
(2.18 \%)\end{array}$ & $\begin{array}{c}2.12 \\
(6.15 \%)\end{array}$ \\
\hline & & 5.5 & $\begin{array}{c}3.04 \\
(1.17 \%)\end{array}$ & $\begin{array}{c}2.08 \\
(3.78 \%)\end{array}$ & $\begin{array}{c}3.04 \\
(1.19 \%)\end{array}$ & $\begin{array}{c}2.08 \\
(3.96 \%)\end{array}$ & $\begin{array}{c}3.03 \\
(1.16 \%)\end{array}$ & $\begin{array}{c}2.07 \\
(3.65 \%)\end{array}$ & $\begin{array}{c}3.04 \\
(1.21 \%)\end{array}$ & $\begin{array}{c}2.08 \\
(4.13 \%) \\
\end{array}$ \\
\hline \multirow[t]{3}{*}{100} & \multirow[t]{3}{*}{4} & 3.5 & $\begin{array}{c}3.02 \\
(0.72 \%)\end{array}$ & $\begin{array}{c}2.04 \\
(2.22 \%)\end{array}$ & $\begin{array}{c}3.02 \\
(0.73 \%)\end{array}$ & $\begin{array}{c}2.05 \\
(2.28 \%)\end{array}$ & $\begin{array}{c}3.02 \\
(0.71 \%)\end{array}$ & $\begin{array}{c}2.04 \\
(2.17 \%)\end{array}$ & $\begin{array}{c}3.02 \\
(0.73 \%)\end{array}$ & $\begin{array}{c}2.05 \\
(2.34 \%)\end{array}$ \\
\hline & & 4.5 & $\begin{array}{c}3.02 \\
(0.51 \%)\end{array}$ & $\begin{array}{c}2.04 \\
(1.86 \%)\end{array}$ & $\begin{array}{c}3.02 \\
(0.51 \%)\end{array}$ & $\begin{array}{c}2.04 \\
(1.90 \%)\end{array}$ & $\begin{array}{c}3.02 \\
(0.51 \%)\end{array}$ & $\begin{array}{c}2.04 \\
(1.83 \%)\end{array}$ & $\begin{array}{c}3.02 \\
(0.52 \%)\end{array}$ & $\begin{array}{c}2.04 \\
(1.94 \%)\end{array}$ \\
\hline & & 5.5 & $\begin{array}{c}3.01 \\
(0.34 \%)\end{array}$ & $\begin{array}{c}2.03 \\
(1.58 \%)\end{array}$ & $\begin{array}{c}3.01 \\
(0.34 \%)\end{array}$ & $\begin{array}{c}2.03 \\
(1.61 \%)\end{array}$ & $\begin{array}{c}3.01 \\
(0.34 \%)\end{array}$ & $\begin{array}{c}2.03 \\
(1.56 \%)\end{array}$ & $\begin{array}{c}3.01 \\
(0.35 \%)\end{array}$ & $\begin{array}{c}2.03 \\
(1.64 \%)\end{array}$ \\
\hline \multirow[t]{3}{*}{150} & \multirow[t]{3}{*}{4} & 3.5 & $\begin{array}{c}3.03 \\
(0.99 \%) \\
\end{array}$ & $\begin{array}{c}2.06 \\
(2.98 \%) \\
\end{array}$ & $\begin{array}{c}3.03 \\
(1.01 \%) \\
\end{array}$ & $\begin{array}{c}2.06 \\
(3.09 \%) \\
\end{array}$ & $\begin{array}{c}3.03 \\
(0.99 \%) \\
\end{array}$ & $\begin{array}{c}2.06 \\
(2.89 \%) \\
\end{array}$ & $\begin{array}{c}3.03 \\
(1.02 \%) \\
\end{array}$ & $\begin{array}{c}2.06 \\
(3.19 \%) \\
\end{array}$ \\
\hline & & 4.5 & $\begin{array}{c}3.02 \\
(0.61 \%)\end{array}$ & $\begin{array}{c}2.05 \\
(2.33 \%)\end{array}$ & $\begin{array}{c}3.02 \\
(0.61 \%)\end{array}$ & $\begin{array}{c}2.05 \\
(2.39 \%)\end{array}$ & $\begin{array}{c}3.02 \\
(0.60 \%)\end{array}$ & $\begin{array}{c}2.05 \\
(2.28 \%)\end{array}$ & $\begin{array}{c}3.02 \\
(0.62 \%)\end{array}$ & $\begin{array}{c}2.05 \\
(2.46 \%)\end{array}$ \\
\hline & & 5.5 & $\begin{array}{c}3.01 \\
(0.31 \%)\end{array}$ & $\begin{array}{c}2.04 \\
(1.87 \%)\end{array}$ & $\begin{array}{c}3.01 \\
(0.31 \%)\end{array}$ & $\begin{array}{c}2.04 \\
(1.91 \%)\end{array}$ & $\begin{array}{c}3.01 \\
(0.31 \%)\end{array}$ & $\begin{array}{c}2.04 \\
(1.84 \%)\end{array}$ & $\begin{array}{c}3.01 \\
(0.31 \%)\end{array}$ & $\begin{array}{c}2.04 \\
(1.95 \%)\end{array}$ \\
\hline \multirow[t]{3}{*}{200} & \multirow[t]{3}{*}{9} & 3.5 & $\begin{array}{c}3.18 \\
(6.06 \%)\end{array}$ & $\begin{array}{c}2.25 \\
(12.71 \%)\end{array}$ & $\begin{array}{c}3.19 \\
(6.53 \%)\end{array}$ & $\begin{array}{c}2.30 \\
(15.0 \%)\end{array}$ & $\begin{array}{c}3.17 \\
(5.73 \%)\end{array}$ & $\begin{array}{c}2.23 \\
(11.38 \%)\end{array}$ & $\begin{array}{c}3.21 \\
(7.02 \%)\end{array}$ & $\begin{array}{c}2.36 \\
(18.05 \%)\end{array}$ \\
\hline & & 4.5 & $\begin{array}{c}3.09 \\
(3.01 \%)\end{array}$ & $\begin{array}{c}2.14 \\
(6.90 \%)\end{array}$ & $\begin{array}{c}3.09 \\
(3.12 \%)\end{array}$ & $\begin{array}{c}2.15 \\
(7.53 \%)\end{array}$ & $\begin{array}{c}3.09 \\
(2.92 \%)\end{array}$ & $\begin{array}{c}2.13 \\
(6.48 \%)\end{array}$ & $\begin{array}{c}3.09 \\
(3.22 \%)\end{array}$ & $\begin{array}{c}2.16 \\
(8.18 \%)\end{array}$ \\
\hline & & 5.5 & $\begin{array}{c}3.05 \\
(1.80 \%) \\
\end{array}$ & $\begin{array}{c}2.09 \\
(4.64 \%) \\
\end{array}$ & $\begin{array}{c}3.06 \\
(1.85 \%) \\
\end{array}$ & $\begin{array}{c}2.09 \\
(4.92 \%) \\
\end{array}$ & $\begin{array}{c}3.05 \\
(1.78 \%) \\
\end{array}$ & $\begin{array}{c}2.09 \\
(4.45 \%) \\
\end{array}$ & $\begin{array}{c}3.06 \\
(1.88 \%) \\
\end{array}$ & $\begin{array}{c}2.10 \\
(5.18 \%) \\
\end{array}$ \\
\hline \multirow[t]{3}{*}{250} & \multirow[t]{3}{*}{8} & 3.5 & $\begin{array}{c}3.03 \\
(1.12 \%)\end{array}$ & $\begin{array}{c}2.06 \\
(2.95 \%)\end{array}$ & $\begin{array}{c}3.03 \\
(1.13 \%)\end{array}$ & $\begin{array}{c}2.06 \\
(3.06 \%)\end{array}$ & $\begin{array}{c}3.03 \\
(1.10 \%)\end{array}$ & $\begin{array}{c}2.06 \\
(2.87 \%)\end{array}$ & $\begin{array}{c}3.03 \\
(1.15 \%)\end{array}$ & $\begin{array}{c}2.06 \\
(3.15 \%)\end{array}$ \\
\hline & & 4.5 & $\begin{array}{c}3.03 \\
(0.87 \%)\end{array}$ & $\begin{array}{c}2.05 \\
(2.45 \%)\end{array}$ & $\begin{array}{c}3.03 \\
(0.88 \%)\end{array}$ & $\begin{array}{c}2.05 \\
(2.52 \%)\end{array}$ & $\begin{array}{c}3.03 \\
(0.86 \%)\end{array}$ & $\begin{array}{c}2.05 \\
(2.39 \%)\end{array}$ & $\begin{array}{c}3.03 \\
(0.89 \%)\end{array}$ & $\begin{array}{c}2.05 \\
(2.59 \%)\end{array}$ \\
\hline & & 5.5 & $\begin{array}{c}3.02 \\
(0.68 \%)\end{array}$ & $\begin{array}{c}2.04 \\
(2.09 \%)\end{array}$ & $\begin{array}{c}3.02 \\
(0.69 \%)\end{array}$ & $\begin{array}{c}2.04 \\
(2.14 \%)\end{array}$ & $\begin{array}{c}3.02 \\
(0.68 \%)\end{array}$ & $\begin{array}{c}2.04 \\
(2.04 \%)\end{array}$ & $\begin{array}{c}3.02 \\
(0.69 \%)\end{array}$ & $\begin{array}{c}2.04 \\
(2.19 \%)\end{array}$ \\
\hline
\end{tabular}

Table 4. Bayes estimates using Jeffreys' prior information for $S E L$ and $B S E L$ function.

\begin{tabular}{|c|c|c|c|c|c|c|c|c|c|c|c|}
\hline \multirow[t]{4}{*}{$n$} & \multirow[t]{4}{*}{$m$} & \multicolumn{10}{|c|}{$\mathrm{TK}$} \\
\hline & & \multirow{2}{*}{\multicolumn{2}{|c|}{ SEL }} & \multicolumn{8}{|c|}{ BSEL } \\
\hline & & & & \multicolumn{2}{|c|}{$w=0.2$} & \multicolumn{2}{|c|}{$w=0.4$} & \multicolumn{2}{|c|}{$w=0.6$} & \multicolumn{2}{|c|}{$w=0.8$} \\
\hline & & $\hat{\alpha}_{S E L}$ & $\hat{\lambda}_{S E L}$ & $\widehat{\alpha}_{B S E L}$ & $\hat{\lambda}_{B S E L}$ & $\hat{\alpha}_{B S E L}$ & $\hat{\lambda}_{B S E L}$ & $\hat{\alpha}_{B S E L}$ & $\hat{\lambda}_{B S E L}$ & $\hat{\alpha}_{B S E L}$ & $\hat{\lambda}_{B S E L}$ \\
\hline 50 & 7 & $\begin{array}{c}2.79 \\
(6.9 \%)\end{array}$ & $\begin{array}{c}1.77 \\
(11 \%)\end{array}$ & $\begin{array}{c}2.83 \\
(5.5 \%)\end{array}$ & $\begin{array}{c}1.82 \\
(9.0 \%)\end{array}$ & $\begin{array}{c}2.88 \\
(4.1 \%)\end{array}$ & $\begin{array}{c}1.86 \\
(6.8 \%)\end{array}$ & $\begin{array}{c}2.92 \\
(2.8 \%)\end{array}$ & $\begin{array}{c}1.91 \\
(4.5 \%)\end{array}$ & $\begin{array}{c}2.96 \\
(1.4 \%)\end{array}$ & $\begin{array}{c}1.96 \\
(2.3 \%)\end{array}$ \\
\hline 100 & 4 & $\begin{array}{c}3.06 \\
(2.1 \%)\end{array}$ & $\begin{array}{c}2.09 \\
(4.9 \%)\end{array}$ & $\begin{array}{c}3.05 \\
(1.6 \%)\end{array}$ & $\begin{array}{c}2.08 \\
(3.9 \%)\end{array}$ & $\begin{array}{c}3.04 \\
(1.2 \%)\end{array}$ & $\begin{array}{c}2.06 \\
(2.9 \%)\end{array}$ & $\begin{array}{c}3.03 \\
(0.8 \%)\end{array}$ & $\begin{array}{c}2.04 \\
(1.9 \%)\end{array}$ & $\begin{array}{c}3.01 \\
(0.4 \%)\end{array}$ & $\begin{array}{c}2.02 \\
(0.9 \%)\end{array}$ \\
\hline 150 & 4 & $\begin{array}{c}3.15 \\
(4.9 \%) \\
\end{array}$ & $\begin{array}{c}2.22 \\
(11 \%)\end{array}$ & $\begin{array}{c}3.12 \\
(3.9 \%) \\
\end{array}$ & $\begin{array}{c}2.18 \\
(8.8 \%)\end{array}$ & $\begin{array}{c}3.09 \\
(2.9 \%) \\
\end{array}$ & $\begin{array}{c}2.13 \\
(6.6 \%)\end{array}$ & $\begin{array}{c}3.06 \\
(1.9 \%) \\
\end{array}$ & $\begin{array}{c}2.09 \\
(4.4 \%) \\
\end{array}$ & $\begin{array}{c}3.03 \\
(0.9 \%) \\
\end{array}$ & $\begin{array}{c}2.05 \\
(2.2 \%) \\
\end{array}$ \\
\hline 200 & 9 & $\begin{array}{c}2.85 \\
(5.1 \%)\end{array}$ & $\begin{array}{c}1.83 \\
(8.5 \%)\end{array}$ & $\begin{array}{c}2.88 \\
(4.1 \%)\end{array}$ & $\begin{array}{c}1.86 \\
(6.8 \%)\end{array}$ & $\begin{array}{c}2.91 \\
(3.1 \%)\end{array}$ & $\begin{array}{c}1.89 \\
(5.1 \%)\end{array}$ & $\begin{array}{c}2.93 \\
(2.0 \%)\end{array}$ & $\begin{array}{c}1.93 \\
(3.4 \%)\end{array}$ & $\begin{array}{c}2.97 \\
(1.0 \%)\end{array}$ & $\begin{array}{c}1.97 \\
(1.7 \%)\end{array}$ \\
\hline 250 & 8 & $\begin{array}{c}3.08 \\
(2.6 \%)\end{array}$ & $\begin{array}{c}2.13 \\
(6.3 \%)\end{array}$ & $\begin{array}{c}3.06 \\
(2.1 \%)\end{array}$ & $\begin{array}{c}2.10 \\
(5.0 \%) \\
\end{array}$ & $\begin{array}{c}3.05 \\
(1.5 \%)\end{array}$ & $\begin{array}{c}2.08 \\
(3.8 \%)\end{array}$ & $\begin{array}{c}3.03 \\
(1.0 \%)\end{array}$ & $\begin{array}{c}2.05 \\
(2.5 \%) \\
\end{array}$ & $\begin{array}{c}3.02 \\
(0.5 \%)\end{array}$ & $\begin{array}{c}2.03 \\
(1.3 \%)\end{array}$ \\
\hline
\end{tabular}




\section{Conclusion}

Theoretical results of the study are explained numerically in the above Tables. Here, MLE's and Bayes estimators based on generated record values are obtained. In Table 2 and Table 3 , we observe that if the value of $c$ increases then we observed that we have the good estimators under different loss functions. From Tables 2, 3, 4 and 5, we see that, the $P E$ of the Bayes estimates under SEL function using extension Jeffreys' prior information and Jeffreys' prior information are more than $P E$ of the Bayes estimates under BSEL function using extension Jeffreys' prior information and Jeffreys' prior information. So we observe that Bayes estimates under BSEL function using extension Jeffreys' prior information and Jeffreys' prior information perform good compared to the $S E L$ function using extension Jeffreys' prior information and Jeffreys' prior information. Moreover, the performances of all the estimators of $B S E L$ function are improved when the value of $\omega$ increases. Mostly, $M L E$ 's have more $P E$ as compare to Bayes estimators under SEL function using extension Jeffreys' prior information and $M L E$ ' $S$ have less $P E$ as compare to Bayes estimators under $S E L$ function using Jeffreys' prior information. Mostly, $M L E$ 's have more $P E$ as compare to Bayes estimators under $G E L$ function using extension Jeffreys' prior information for both negative and positive values of $k$ and $M L E$ 's have less $P E$ as compare to Bayes estimators under GEL function using Jeffreys' prior information for both negative and positive values of $k$. Finally, we observe that Bayes estimators under extension Jeffreys' prior information perform good as compared to the Bayes estimators under Jeffreys' prior information.

\section{Acknowledgment}

The authors thank the referees for their constructive and useful comments and suggestions regarding the first version of this manuscript, which led to this improved version.

\section{References}

1. Ahmed, E. A. (2014). Bayesian estimation based on progressive Type-II censoring from two-parameter bathtub-shaped lifetime model: an Markov Chain Monte Carlo approach. Journal of Applied Statistics, 41, 752-768.

2. Ahsanullah, M. (2004). Record Values-Theory and Applications. University Press of America.

3. Arnold, B. C., Balakrishnan, N. and Nagaraja, H. N. (1998). Records. John Wiley and Sons.

4. Bernardo, J. M. (1979). Reference posterior distributions for Bayesian inference. Journal of the Royal Statistical Society, B(2), 113-147.

5. Box, G. E. P and Tiao, G. C. (1973). Bayesian Inference in Statistical Analysis. John Wiley and Sons.

6. Calabria, R. and Pulcini, G. (1996). Point estimation under asymmetric loss functions for left-truncated exponential samples. Communications in StatisticsTheory and Methods, 25, 585-600.

7. Chandler, K. N. (1952). The distribution and frequency of record values. Journal of the Royal Statistical Society, B(14), 220-228.

8. Dey, D. K. and Liu, P. S. L. (1992). On comparison of estimators in a generalized life model. Microelectronics Reliability, 32, 207-221. 
9. Faizan, M. and Sana (2018): Bayesian estimation and prediction for Chen distribution based on upper record values. Journal of Mathematics and Statistical Science, 4(6), 235-243.

10. Gencer, G. and Saraçoğlu, B. (2016). Comparison of approximate Bayes estimators under different loss functions for parameters of odd Weibull distribution. Journal of Selçuk University Natural and Applied Science, 5(1), 18-32.

11. Guure, C. B., Ibrahim, N. A. and Adam, M. B. (2013). Bayesian inference of the Weibull model based on interval-censored survival data. Computational and Mathematical Methods in Medicine, 2013, Article ID 849520.

12. Haq, A. and Dey, S. (2011). Bayesian estimation of Erlang distribution under different prior distributions. Journal of Reliability and Statistical Studies, 4, 1-30.

13. Jeffreys', H. (1946). An invariant form for the prior probability in estimation problems. Proceedings of the Royal Society of London, Series A, Mathematical and Physical Sciences, 186, 453-461.

14. Jeffreys', H. (1961). Theory of Probability (3rd ed.). Oxford: Oxford University Press.

15. Kumar, D., Dey, T. and Dey, S. (2017). Statistical inference of exponentiated moment exponential distribution based on lower record values. Communications in Mathematics and Statistics, 5, 231-260.

16. Kass, R. E. (1990). Data-translated likelihood and Jeffreys' rules. Biometrika, 77, 107-114.

17. Kass, R. E. (1989). The geometry of asymptotic inference. Statistical Science, 4, 188-219.

18. Kayal, T., Tripathi, Y. M., Singh, D. P. and Rastogi, M. K. (2017). Estimation and prediction for Chen distribution with bathtub shape under progressive censoring. Journal of Statistical Computation and Simulation, 87, 348-366.

19. Lavanya, A. and Alexander, T. L. (2016). Estimation of parameters using Lindley's method. International Journal of Advanced Research, 4, 1767-1778.

20. Legendre, A. M. (1805). Nouvelles méthodes pour la determination des orbites des comètes. F. Didot.

21. Jung, M. and Chung, Y. (2018). Bayesian inference of three-parameter bathtubshaped lifetime distribution. Communications in Statistics: Theory and Methods, 47, 4229-4241.

22. Pandey, R. and Kumari, N. (2018a). Bayesian analysis of power generalized Weibull distribution. International Journal of Applied and Computational Mathematics, 4, 141.

23. Pandey, R. and Kumari, N. (2018b). Bayesian estimation for ISB p-dim Rayleigh distribution under type-II censored data using Lindley's approximation. World Wide Journal of Multidisciplinary Research and Development, 4, 207-211.

24. Nadarajah, S. and Haghighi, F. (2011). An extension of the exponential distribution. Statistics, 45, 543-558.

25. R Core Team (2016). R: A language and environment for statistical computing. R Foundation for Statistical Computing, Vienna, Austria. URL https://www.Rproject.org/.

26. Selim, M. A. (2012). Bayesian estimations from the two-parameter bathtub-shaped lifetime distribution based on record values. Pakistan Journal of Statistics and Operation Research, 8, 155-165. 
27. Selim, M. A. (2018). Estimation and prediction for Nadarajah-Haghighi distribution based on record values. Pakistan Journal of Statistics, 34, 77-90.

28. Singh, U., Singh, S. K. and Yadav, A. S. (2015). Bayesian estimation for extension of exponential distribution under progressive Type-II censored data using Markov Chain Monte Carlo Method. Journal of Statistics Applications and Probability, 4, 275-283.

29. Singh, S. K., Singh, U., Kumar, M. and Vishwakarma, P. K. (2014). Classical and Bayesian inference for an extension of the exponential distribution under progressive type-II censored data with binomial removals. Journal of Statistics Applications and Probability Letters, 3, 75-86.

30. Tierney, L., and Kadane, J. B. (1986). Accurate approximations for posterior moments and marginal densities. Journal of the American Statistical Association, 81, 82-86. 